\title{
Data-aided Iterative Algorithms for Linearizing IM/DD Optical Transmission Systems
}

Hu, Shaohua; Zhang, Jing; Tang, Jianming; Jin, Wei; Giddings, Roger; Qiu, Kun

\section{Journal of Lightwave Technology}

DOI:

10.1109/JLT.2021.3063689

Published: 01/05/2021

Peer reviewed version

Cyswllt i'r cyhoeddiad / Link to publication

Dyfyniad o'r fersiwn a gyhoeddwyd / Citation for published version (APA):

Hu, S., Zhang, J., Tang, J., Jin, W., Giddings, R., \& Qiu, K. (2021). Data-aided Iterative Algorithms for Linearizing IM/DD Optical Transmission Systems. Journal of Lightwave Technology, 39(9), 2864 - 2872. https://doi.org/10.1109/JLT.2021.3063689

\footnotetext{
Hawliau Cyffredinol / General rights

Copyright and moral rights for the publications made accessible in the public portal are retained by the authors and/or other copyright owners and it is a condition of accessing publications that users recognise and abide by the legal requirements associated with these rights.

- Users may download and print one copy of any publication from the public portal for the purpose of private study or research.

- You may not further distribute the material or use it for any profit-making activity or commercial gain

- You may freely distribute the URL identifying the publication in the public portal ?
}

Take down policy

If you believe that this document breaches copyright please contact us providing details, and we will remove access to the work immediately and investigate your claim. 


\title{
Data-aided Iterative Algorithms for Linearizing IM/DD Optical Transmission Systems
}

\author{
Shaohua Hu, Jing Zhang, Jianming Tang, Member, IEEE, Wei Jin, \\ Roger Giddings, Member IEEE, Kun Qiu
}

\begin{abstract}
DSP-enhanced intensity-modulation direct-detection (IM/DD) systems can support up to $56 \mathrm{~Gb} / \mathrm{s}$ over $100 \mathrm{~km}$ signal transmissions at $\mathrm{C}$-band. To achieve higher data rates and longer transmission distances, we propose data-aided iterative algorithm (DIA) and decision-directed DIA (DD-DIA) to digitally mitigate signal-signal beating interference (SSBI) without requiring any modifications to physical layer structures. DIA utilizes pilot symbols with uniformly spaced insertions to relax the modified Gerchberg-Saxton (G-S) algorithms that suffer from the local optimum problem. To further improve the symbol error rate (SER) performance and convergence speed, DD-DIA introduces a decision process to generate pseudo-pilots. We numerically compare DIA with other algorithms and find that DIA can recover signals subject to large fiber dispersions corresponding to which conventional IA and Volterra filter (VF) fail, while DD-DIA significantly accelerates the convergence speed and improves the reconstruction performance compared with DIA, it can support 100-Gb/s PAM4 over 400-km IM/DD transmissions within just 50 iterations. Two orders of magnitude reductions in SER is observed for $100 \mathrm{~Gb} / \mathrm{s}$ PAM4 signal transmission over $100-\mathrm{km}$ SSMFs. Compared with conventional IA, the proposed techniques have higher convergence speeds, better global optimum features and large tolerances to physical model errors. In particular, DD-DIA results in larger optical signal-to-noise ratio (OSNR)/ received optical power (ROP) improvement, higher transmission capacities and less computational complexity. DD-DIA is a promising algorithm for efficiently reconstructing 2-dimensional optical field for conventional IM/DD optical transmission systems.

Index Terms-Optical communication, digital signal processing, IM/DD system, field reconstruction.
\end{abstract}

\section{INTRODUCTION}

$\mathrm{W}$ ITH the ever exponentially increasing requirement for long-haul and high-speed communications, electronic dispersion compensation (EDC) has matured and been

The manuscript was received on xxx. This work was supported in part by the DESTINI project funded by the ERDF under the SMARTExpertise scheme, the DSP Centre funded by the ERDF through the Welsh Government, in part by the NSFC (61871082 and 61420106011), the state Key Laboratory of Advanced Optical Communication Systems and Networks, Shanghai Jiao Tong University (2020GZKF014), Fundamental Research Funds for the Central Universities (ZYGX2019J008) and the program of China Scholarships Council (No. 201906070011). (Corresponding author:Jing Zhang).

Shaohua Hu, Jing Zhang, and Kun Qiu are with the Key Laboratory of Optical Fiber Sensing and Communications (Education Ministry of China), School of Communication and Information Engineering, University of Electronic Science and Technology of China, Chengdu, Sichuan 611731, China (e-mail: hshhfmy@std.uestc.edu.cn; $\quad$ zhangjing1983@uestc.edu.cn; kqiu@uestc.edu.cn).

Jianming Tang, Wei Jin and Roger Gidding are with the School of Electronic Engineering, Bangor University, Bangor, LL57 1UT, UK. (email: j.tang@bangor.ac.uk; w.jin@bangor.ac.uk; r.p.giddings@bangor.ac.uk) practically deployed in coherent optical transmission systems [1]. However, for intensity modulation and direct detection (IM/DD) optical transmission systems that are widely implemented for short or medium reach communications scenarios, the available EDC technique cannot be directly applied to compensate for fiber chromatic dispersion because of signal-signal beating interference (SSBI) induced by square-law photon detection. It is difficult to compensate for the SSBI effect perfectly especially for cases where a large accumulated fiber dispersion occurs. To fully reconstruct the optical field in the receiver end, significant modifications to receiver configurations have been undertaken by utilizing schemes such as the single sideband (SSB) techniques [2], Kramers-Kronig reception [3], delay interferometers [4], Stokes vector receivers and self-homodyne detection[5]. However, all of the abovementioned schemes change the physical layer structure of a conventional IM/DD transmission system. In addition, at the transmitter side, dual-drive Mach-Zehnder modulators (DD-MZMs) [2], I/Q-modulators [6] or optical filters [4] are also required to achieve optical SSB modulation. As a result, a direct optical field recovery in an IM/DD system without changing the physical layer system structure is highly expected for practical deployment.

To enhance the transmission performance but without any physical structure modifications to conventional IM/DD optical transmission systems, several digital signal processing (DSP)-based performance enhancement algorithms have been presented to combat the effects including $\mathrm{CD}$, the equalization-induced noise enhancement and transceiver nonlinearities. These techniques include Volterra equalization [7], Tomlinson-Harashima precoding [8] and FDE-MLSE [9]. Regardless of their algorithm complexity, these techniques can support up to $84-\mathrm{Gb} / \mathrm{s}$ over $80-\mathrm{km}$ [9] or $56-\mathrm{Gb} / \mathrm{s}$ over $100-\mathrm{km}$ [10] optical transmissions at C-band.

Recently, an iterative field retrieval algorithm originating from the G-S algorithm developed for optical imaging shows good potential for further improving the signal transmission capacity of the IM/DD optical transmission systems. In previously published work, the modified G-S algorithms can be applied at the transmitter [11] and the receiver [10, 12] for pre-emphasis and post-compensation, respectively. In the algorithms, the received optical current is regarded as the image plane, and the transmitted optical signal is regarded as the object plane where its phase is determined by pre-determined modulator/system characteristics including the chromatic dispersion parameter, the fiber length and the characteristics of the intensity modulator. To minimize the errors associated with 
the square-law detected signal at the receiver, optimizations of the received optical field should be performed to ensure good matching between the estimated optical field and the real optical field after transmitting through the physical transmission system. This is referred to as the local-optimum problem, which causes the first derivative of the convergence curve to approach zero even when the errors are still large. This local-optimum problem is more significant for large accumulated dispersion cases. To address such a problem, in a recently published paper [13], with the help of training symbols and a dispersion device, the G-S algorithm is used to reconstruct the complex optical signal iteratively. However, the algorithm requires an extra dispersive device and two PDs, leading to the increase in the receiver complexity. In addition, to reduce the estimated errors, the algorithm reported in [13] also needs an "escape" operation that may require thousands of iterations.

In order to efficiently linearize the IM/DD optical transmission systems in a low-cost way, in this paper, we introduce pilot symbols into the transmitted frames in the receiver to accelerate the convergence of the conventional iterative algorithms. The proposed technique is termed data-aided iterative algorithm (DIA), which can recover the optical field and transmitted data received by only one photodiode even at the presence of severe CD-induced distortions that lead to the operation failure of the first three-order Volterra equalization and conventional iterative G-S algorithms. Through numerical simulations, the effectiveness of the proposed DIA technique is demonstrated in terms of improving the OSNR/ROP, signal transmission capacity and convergence acceleration. Our simulations show that for $100 \mathrm{~Gb} / \mathrm{s}$ PAM4 signal transmissions over $50 \mathrm{~km} \mathrm{SSMF}$ at an OSNR of $43 \mathrm{~dB}$ and a ROP of $-4 \mathrm{dBm}$, more than 5-dB ROP improvement and up to 4-dB OSNR improvement are obtainable when a DIA overhead as low as $6.7 \%$ is introduced. It is also shown that the increase in the pilot symbol repetition rate is beneficial to both the convergence speed and the reconstructed signal performance.

Furthermore, a modified DIA technique called decision directed DIA (DD-DIA) is also proposed in this paper, which uses randomly selected symbols to enrich the pilot collection and increase the repetition rate of the pilot symbols. With the help of the pilot symbols from DIA and the decided symbols from the decision process, the IM/DD optical transmission systems can support 100-Gb/s 400-km optical fiber transmissions at C-band. Compared with the DIA technique, the symbol decision in DD-DIA contributes symbol error reductions by two orders of magnitude for $100-\mathrm{Gb} / \mathrm{s}$ over 100-km PAM4 transmissions. The symbol decision in DD-DIA enables further significant performance improvements when DIA reduces the SER to $1 \times 10^{-1}$. A good DD-DIA convergence is obtainable within just 50 iterations. Furthermore, the decision process offers DIA a large tolerance to channel parameter estimation errors.

This paper is organized as follows. Section II presents the principles of DIA and the pilot allocation method. In Section III, we verify DIA's performance in improving OSNRs/ROPs, transmission distances/data rates and convergence performance, as well as the tolerance to channel physical-model estimation errors. In Section IV, we introduce the DD-DIA technique and explore its performance enhancement compared with DIA. Finally, the advantages of our proposed algorithm are summarized briefly in Section V.

\section{PRINCIPLES}

To describe the signal reconstruction principles, we consider a conventional IM/DD optical transmission system. At the transmitter side, the optical field after an intensity modulator can be expressed as

$$
\underline{E_{T x}(t)=\sqrt{A+d(t)} \mathrm{e}^{j\left[\omega_{0} t+\varphi_{\text {Chip }}(t)\right]},}
$$

where $d(t)$ is the electrical driving signal, $A$ and $\omega$ are the bias current of the modulator's driving signal and the central angular frequency of the optical signal. The intensity modulator's chirp-induced additional phase, which is related to the optical power $P(t)$, can be expressed as[14, 15],

$$
\varphi_{\text {Chip }}(t) \cong \frac{\alpha}{4 \pi} \int\left[\frac{1}{P(t)} \frac{d P(t)}{d t}+\kappa P(t)\right] d t=\frac{\alpha}{4 \pi}[\kappa P(t) t+\ln P(t)]
$$

The linewidth enhancement factor $\alpha$ and the adiabaticchirp coefficient $\kappa$ of a commercially available DML are about 2-5 and $10-15 \mathrm{GHz} / \mathrm{mW}$ respectively, whilst an EML can have $\kappa=0$. An ideal intensity modulator has $\varphi_{\text {Chir }}(t)=0$. After fiber transmission, the complex spectrum of the received optical field can be expressed as[16].

$$
E_{R x}(f)=E_{T x}(f) H_{C D}(f), \quad H_{C D}(f)=\mathrm{e}^{-j \pi D \frac{\lambda^{2}}{c} L f^{2}},
$$

where $D, \lambda, c, L$, and $f$ represent the fiber dispersion, the central wavelength, the velocity of light, the transmission distance, and the analogue frequency, respectively. $H_{C D}(f)$ is the frequency-domain transfer function of the dispersive fiber. The PD detected signal can be expressed in Eq. (4) which contains linear terms after Taylor expansion[16],

$$
I_{\mathrm{o}}(t) \approx A+d(t) \otimes \operatorname{Re}\left[h_{C D}(t)\right]=A+d(t) \otimes \mathbf{F}^{-1}\left[\cos \left(\pi D \frac{\lambda^{2}}{c} L f^{2}\right)\right]
$$

where $\operatorname{Re}\left[\square, \mathbf{F}^{-1}\right.$ and $\otimes$ represent the real part of the complex signal, the inverse Fourier transform and convolution operations. The DC component $A$ can be easily filtered out after the optical-to-electrical conversion. For small signal analysis, the approximated power fading consisting of the intensity modulator, fiber transmission and a detector can be described by power spectrum density $[15]|H(f)|^{2}$ as Eq. (5),

$$
\begin{gathered}
|H(f)|^{2}=\left(1+\alpha^{2}\right) \cos ^{2}\left(2 \pi^{2} f^{2} \beta_{2} L-\tan ^{-1} \alpha\right) \\
+\frac{\alpha^{2} \kappa^{2} A^{2}}{4 \pi^{2} f^{2}} \sin ^{2}\left(2 \pi^{2} f^{2} \beta_{2} L\right)
\end{gathered}
$$

where $\beta_{2}$ is the group-velocity dispersion coefficient. The expression of SSBI can be found in many research papers, 


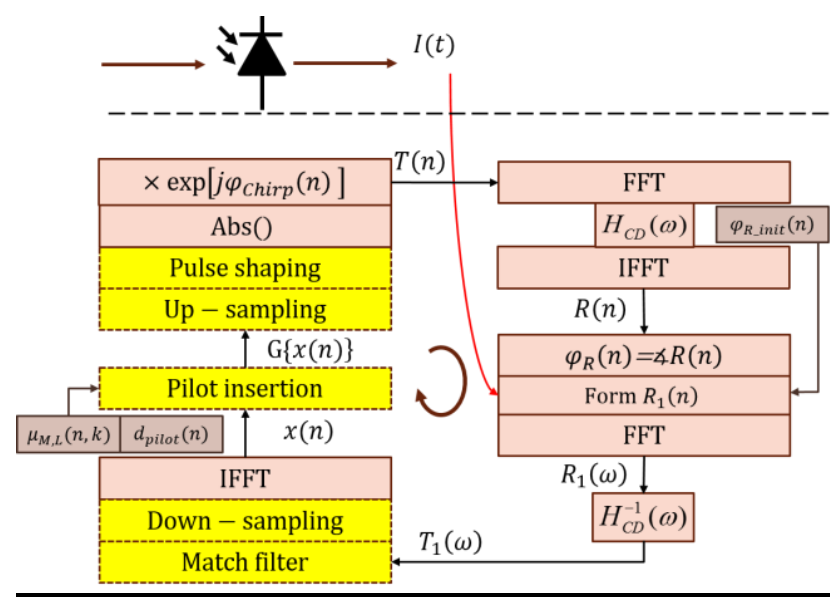

Fig. 1 The workflow of data-aided iterative algorithm for signal reconstruction.

which can be Taylor-expanded to higher orders and difficult to be compensated by traditional equalization [17].

To process the signal sequence after an ADC in the receiver, we resample the signal by 2 samples per symbol. Assuming the signal is perfectly frame-synchronized, the DIA can be applied following the procedures illustrated in Fig. 1. The algorithm starts at the right hand of Fig.1, namely, the initialization of the phase $\varphi_{R}(n)$ can be set by,

$$
\varphi_{R}(n)=\varphi_{R_{-} i n i t}(n)=2 \pi n / L_{S i g},
$$

where $n$ is the time index and $L_{S i g}$ is the sequence length. The initialization is not unique, which imposes negligible impacts on the linearization performance only if it is a slow time variant. It should be noted that random initializations should be avoided since they result in performance deteriorations. The calculated optical field before the PD $R_{1}(n)=\sqrt{A+I\left(n T_{s}\right)} \exp \left[\mathrm{j} \varphi_{R}(n)\right]$ is then formed and back-propagated in the digital form of Eq. (7),

$$
T_{1}(f)=R_{1}(f) H_{C D}^{-1}(f)=\mathbf{F}\left[\sqrt{A+I(t)} \mathrm{e}^{j \varphi_{R}(t)}\right] \mathrm{e}^{j \pi D \frac{\lambda^{2}}{c} L f^{2}},
$$

where $\varphi_{R}(t)$ is the phase signal of the optical field propagated through the fiber._ $I(t)$ is the detected optical current, which is proportional to the optical intensity injected into the $\mathrm{PD}$. In the digital domain, an analogue frequency $f$ can be replaced with a digital angular frequency $\omega=2 \pi f T_{s}$ where $T_{s}$ is the sampling interval. $T_{1}(\omega)$ is match-filtered and down-sampled to the baud rate and is subject to the pilot-symbol insertion, as expressed in Eq. (8)

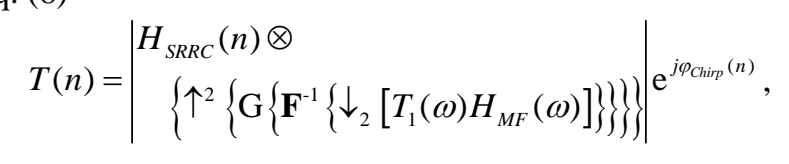

where $\| H_{S R R C}, H_{M F}, \mathbf{F}, \uparrow^{N}, \mathrm{G}$, and $\downarrow_{M}$ represent the modulus value of a complex signal, square-root raise cosine filtering, match filtering, discrete Fourier transform, $N \times$ up-sampling, pilot symbol insertion operation and $M \times$ down-sampling, as shown in the left hand of Fig. 1. Therein, the pilot symbol allocation strategy described in $\mathrm{G}\{x(n)\}$, is expressed as,

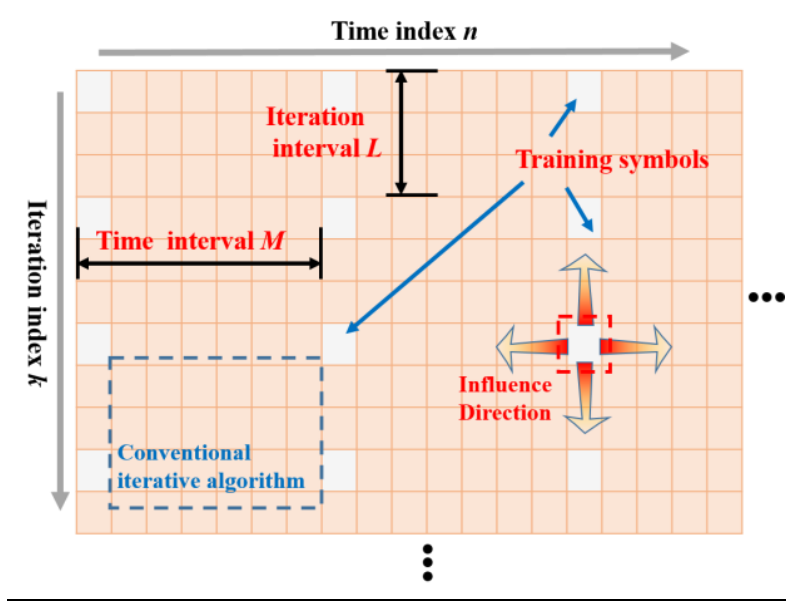

Fig. 2. The diagram of the training strategy of the DIA.

$$
\left\{\begin{array}{l}
\mathrm{G}\{x(n)\}=\mu_{M, L}(n, k) d_{\text {pilot }}(n)+\left[1-\mu_{M, L}(n, k)\right] x(n) \\
\mu_{M, L}(n, k)= \begin{cases}1, & \text { for } \bmod (n, M)=0 \& \bmod (k, L)=0, \\
0, & \text { otherwise }\end{cases}
\end{array}\right.
$$

where $\mu_{M, L}(n, k)$ is the flag representing whether we insert pilot symbol to $T_{1}(t)$ at a specific time and iteration slots. $M$, $\underline{L, n \text {, and } k \text { represent the time interval, the iteration interval, the }}$ time index and the iteration index respectively, as shown in Fig. 2, in which the following parameters are taken: $M=7, L=3$ and an overhead of $1 / M \times 100 \%=14.28 \%$. The pilot insertion operation is only enabled at the 1-st, 4-th, 7-th .... cycles of iteration. For the remaining iterations, Eq. (8) can be simplified as

$$
T(n)=\left|\mathbf{F}^{-1}\left[T_{1}(\omega)\right]\right| \mathrm{e}^{j \varphi_{\text {Chip }}(n)},
$$

which indicates the degeneration from the DIA to the conventional IA. The pilot symbol helps the further convergence at neighboring symbols and iterations.

Then the newly constructed signal is forward-propagated through the fiber according to Eq. (3) in order to obtain $R(n)=\mathbf{F}^{-1}\left\{H_{C D}(\omega) \mathbf{F}[T(n)]\right\}$. The angle part of $R(n)$ is then abstracted by $\varphi_{R}(n)=\square R(n)$, which is regarded as an estimated phase of the received optical field. Both $I(t)$ and $\varphi_{R}(n)$ are used to generate a new receiver-side optical field. The above describes the procedures associated with one iteration of the proposed DIA technique.

From the above descriptions, it can be seen that the main differences between the proposed technique and the previously published techniques are: a training sequence is added into the transmitted sequence according to the pilot allocation regular to significantly improve the performance of the optical field phase retrieval in spite of slight computational complexity growth caused by pulse shaping and match-filtering for pilot insertion. The match filtering also contributes to the performance improvement because it shapes the RF spectrum and filters out the out-of-band signal from iterative calculations.

Figure 3(a) shows the convergence curves of some specific time indexes of DIA and conventional IA. The DIA causes the output symbol amplitudes to fluctuate towards the converged value more rapidly. Moreover, some wrongly calculated amplitudes are corrected by DIA. From Fig. 3(b), we find the 

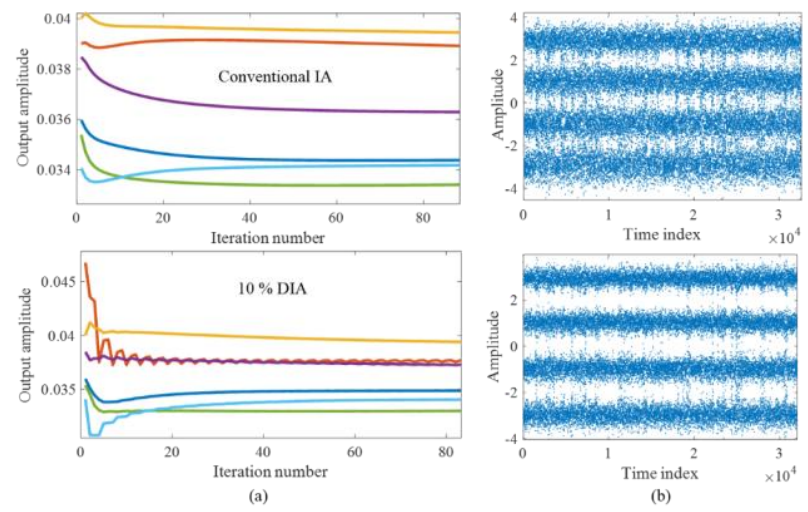

Fig. 3. Comparison of DIA and conventional IA for a $150 \mathrm{~Gb} / \mathrm{s}$ PAM4 signal transmitted over $20 \mathrm{~km} \mathrm{SSMF}$. The OSNR and ROP are $52 \mathrm{~dB}$ and 0 $\mathrm{dBm}$ respectively. (a) The curves of the calculated values by DIA and conventional IA at specific time slots; (b) the scatterplots of DIA and conventional IA after 100 times iteration.

quality of the scatterplot of the PAM4 sequence recovered by DIA is improved considerably.

Note that we do not introduce the "escape" operation to eliminate the local optimum problem, this shortens the execution time and reduces the computational complexity. Furthermore, we can introduce pseudo pilots to further enhance the DIA performance, which can be regarded as another novelty of the proposed technique, as discussed in Section IV.

\section{SimUlations AND DISCUSSIONS}

To verify the performance improvement of the proposed DIA algorithm in DSB IM/DD optical transmission systems, we conduct numerical simulations using VPI, where a single carrier PAM4 signal is transmitted. Fig.4 and Table 1 show the simulation setup and device parameters of the PAM4 DSB IM/DD system.

At the transmitter, a PRBS data sequence is mapped into a PAM4 symbol sequence, which is then up-sampled by a factor of 2. The square-root raised cosine (SRRC) filter with a roll-off factor of 0.1 is applied to the up-sampled sequence to improve the spectral efficiency and partially illuminate CD-induced signal distortions. The electrical-to-optical (E/O) conversion is realized by a Mach-Zander modulator (MZM) biased at $\mathrm{V}_{\pi} / 4$, where the input continuous optical waveform operates at a wavelength of $1550.00 \mathrm{~nm}$ and has a linewidth of $100 \mathrm{kHz}$. The driving voltage amplitude of the RF input to the MZM is $0.4 \mathrm{~V}$.

To compare the performance between DIA, conventional IA, and the first 3 order Volterra nonlinear equalization, the baud rate is set to be 50 GBaud, and hence the output optical signal from the MZM has a $50 \mathrm{GHz}$ bandwidth and a $100 \mathrm{~Gb} / \mathrm{s}$ data rate. An ideal power-controlled EDFA is deployed after the MZM to ensure that the launch power is fixed at $-2 \mathrm{dBm}$. The fiber length is $50 \mathrm{~km}$ with a dispersion parameter of 16 $\mathrm{ps} / \mathrm{nm} / \mathrm{km}$, which is identical to the commercial G. 652 fiber. An optical AWGN source loads noise to the optical signal transmitted through the fiber in order to adjust optical signal's OSNR from 40 to $52 \mathrm{~dB}$. The noise is calculated within a spectral resolution of $0.1 \mathrm{~nm}$. At the receiver, to control the received optical power, a pre-amplifier is employed before the

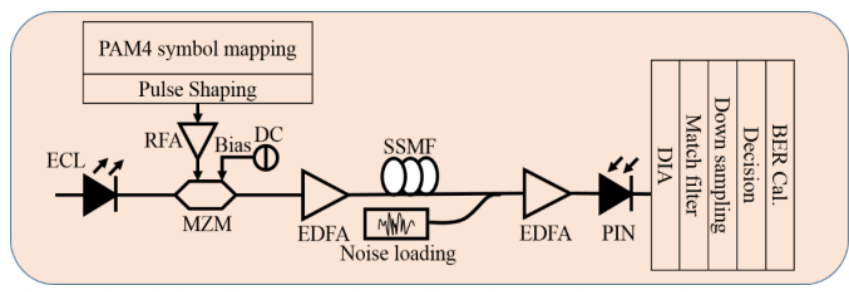

Fig. 4. Simulation diagram and DSP flowchart of the transceiver.

PIN photodiode where thermal noise and shot noise is considered. After optical-to-electrical $(\mathrm{O} / \mathrm{E})$ conversion in the receiver, the electrical current is sampled at a speed of 100 GS/s. The receiver side DSP includes DIA, match filtering, down sampling, and maximum likelihood symbol decision. It should be noted that for cases where the dark current and thermal noise of a PD are higher than those used in the simulations, the extra channel noises just give rise to the increment of SERs at the low ROP ends, and their impacts on the SSBI cancellation performance degradations are negligible.Note, the dark current and thermal noise of PD in practice would be larger than the simulation. This can be regarded as an extra of the channel noise, which may simply lead to the increment of SER at the low end of ROP, however, it eontributes little to the performance degradation in the SSBI eancellation.

TABLE 1. SIMULATION PARAMETERS

\begin{tabular}{ccc} 
& TABLE 1. SIMULATION PARAMETERS \\
\hline \hline & Raw bit rate & $100 \mathrm{~Gb} / \mathrm{s}$ \\
System & Modulation format & PAM4 \\
parameter & SRRC rolling factor & 0.1 \\
& Fiber length & $50 \mathrm{~km}$ \\
& Dispersion parameter & $16 \mathrm{ps} / \mathrm{nm} / \mathrm{km}$ \\
& Central wavelength & $1550 \mathrm{~nm}$ \\
\hline \multirow{2}{*}{ Modulator: } & $\mathrm{V}_{\pi}$ & $10 \mathrm{~V}$ \\
MZM & Bias Voltage & $2.5 \mathrm{~V}$ \\
& Driving amplitude & $0.4 \mathrm{~V}$ \\
\hline \multirow{3}{*}{ PD:PIN } & Dark current & $0 \mathrm{~A}$ \\
& Thermal noise & $10 \times 10^{-12} \mathrm{~A} / \mathrm{Hz}^{1 / 2}$ \\
& Responsivity & $1 \mathrm{~A} / \mathrm{W}$ \\
\hline \hline
\end{tabular}

\section{A. Transmission performance improvement}

The SER versus OSNR and ROP performances are plotted in Fig. 5, where the performances of three algorithms including DIA, conventional IA and Volterra filter (VF) are shown to demonstrate the performance improvement of DIA. The VF with first three orders uses the RLS algorithm for fast convergence, which has been demonstrated outperforming FFE for system linearization [18]. For the DIA case, numerical simulations are undertaken for $25 \%, 10 \%$, and $6.7 \%$ training overheads. The time intervals are 4,10 , and 15 . The iteration intervals are kept at every 3 symbols, i.e., $L=3$ corresponding to Eq. (9).

It is shown that the widely-studied Volterra equalization with 100,11 , and 5 taps corresponding to the $1^{\text {st }}, 2^{\text {nd }}$, and $3^{\text {rd }}$ order, cannot recover the signal, while conventional IA can just reduce the SER of the recovered signal to a level of lower than the $20 \%$ FEC limit. When using the training pilot, the SER decreases significantly compared with conventional IA. There are 4-dB OSNR improvement and larger than 5-dB ROP improvement are achieved by $6.7 \%$ overhead DIA. When the 


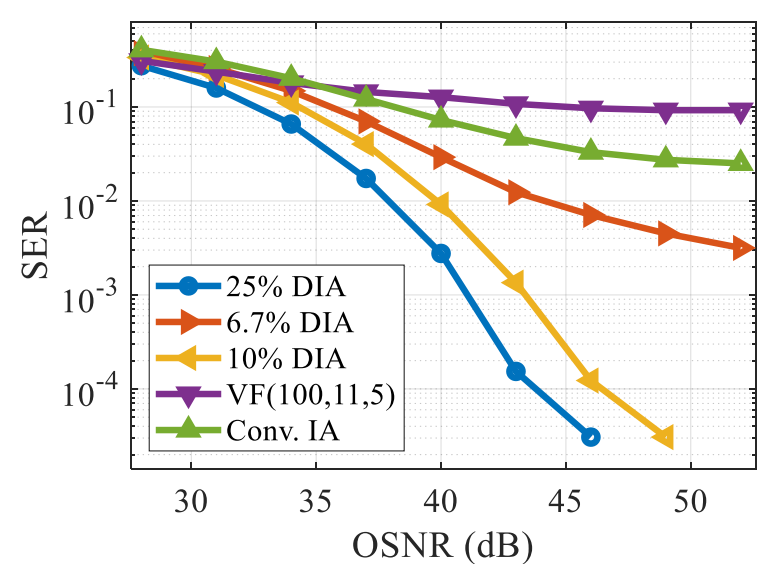

(a)

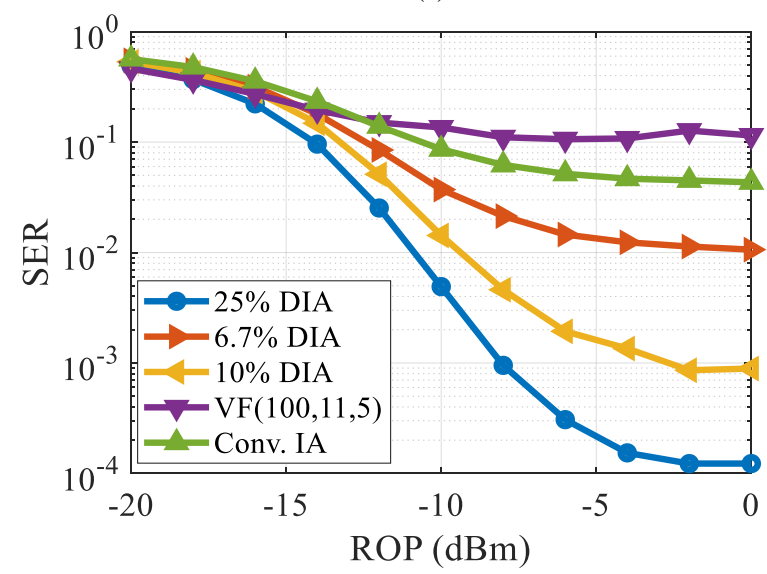

(b)

Fig. 5. The symbol error rate versus (a) OSNR $(\mathrm{ROP}=-4 \mathrm{dBm})$ and (b) ROP $(\mathrm{OSNR}=43 \mathrm{~dB})$ for different algorithms. $x \%$ DIA, data-aided iterative algorithm with $x \%$ training overhead; $\mathrm{VF}(a, b, c)$, Volterra filter

overhead increases to $10 \%$, the $7 \%$ FEC limit can be reached at an OSNR of $43 \mathrm{~dB}$ but without showing any sign of an error floor, as shown in Fig. 5(a). Meanwhile, the SER can be improved by more than 1 order of magnitude at a ROP of -8 $\mathrm{dBm}$. As the accumulated dispersion is stronger at C-band for the $100-\mathrm{Gb} / \mathrm{s}$ optical double-sideband PAM4 signal, it results in stronger SSBI. This is which is-a dominant performance the dominated limitation factor. For further performance improvements, VF should be deployed with hybrid techniques with much llarger memory lengths [19], while the DIA can be upgraded to the DD-DIA with higher linearization capability by simply using a decision process, as detailed illustrated in Section IV in detail.

\section{B. Capacity Improvement}

As discussed in III.A, the DIA-induced gains in OSNR and
ROP can also result in a signal transmission capacity improvement. To explore the DIA-enabled maximum achievable signal transmission capacity of the DSB IM/DD optical transmission system, we transmit the PAM4 signals of different data rates over various fiber lengths, then we evaluate the system performance in terms of SNR versus fiber length for different data rates. The results are plotted in Fig. 6, where DIA's overheads of $6.7 \%, 10 \%$ and $20 \%$ are considered in Fig.6(a), Fig.6(b) and Fig.6(c), respectively. In simulating Fig.6, fixed OSNRs of $43 \mathrm{~dB}$ and fixed ROPs of $-4 \mathrm{dBm}$ are adopted.

Fig.6 shows that for each signal data rate, its SNR of the recovered signal increases with increasing training overhead. For $6.7 \%$ DIA, $80 \mathrm{~Gb} / \mathrm{s}$ can be recovered after $400 \mathrm{~km} \mathrm{SSMF}$ transmissions at the $20 \%$ FEC limit, giving rise to a signal bit rate and distance product of $80 \mathrm{~Gb} / \mathrm{s} \times 400 \mathrm{~km} \times(1-6.7 \%)=7.464$ $\times 10^{4} \mathrm{~Gb} / \mathrm{s} \cdot \mathrm{km}$. While for a $20 \%$ DIA, $150 \mathrm{~Gb} / \mathrm{s}$ can be recovered after $150 \mathrm{~km}$ SSMF transmissions at the $20 \%$ FEC limit, giving rise to a signal bit rate and distance product of 150 $\mathrm{Gb} / \mathrm{s} \times 150 \mathrm{~km} \times(1-20 \%)=1.8 \times 10^{4} \mathrm{~Gb} / \mathrm{s} \cdot \mathrm{km}$. Such DIA-enabled signal reconstruction performances show huge improvements in comparison with the previously published transmission record of $5.6 \times 10^{3} \mathrm{~Gb} / \mathrm{s} \cdot \mathrm{km}$ [12]. This confirms the DIA's capability of linearizing IM/DD optical transmission systems and cancelling the major SSBI effect associated with IM/DD optical transmission systems. It can also be seen in Fig. 6 that the SNR reduction becomes relatively flattened when the SSMF lengths are beyond $40 \mathrm{~km}$. The SNR curve fluctuations are caused by dispersion-induced frequency selective power fading, which is a function of accumulative dispersion.

It should also be noted that although the proposed DIA algorithm with the contemporary training strategy outperforms all other algorithms, it is unable to fully compensate for the CD effect. In Section IV, we show a modification to DIA to further improve the IM/DD system performance.

\section{Convergence Performance}

Since the DIA reconstructs the signal by iterations, the iteration number is thus an important measure of its computational complexity. To explore the DIA's convergence speed, without the loss of generosity, we consider a case where $100 \mathrm{~Gb} / \mathrm{s}$ PAM 4 signals are transmitted over a 50-km SSMF.

In Fig.7 we sketch the SNRs of the recovered signals as a function of iteration number for different ROPs, OSNRs and training overheads. From Fig.7 it is seen that decreasing the time interval of the training pilot can accelerate the algorithm convergence speed, but the tradeoff should be considered between the accelerated convergence speed and net data rate. For $20 \%$ DIA, 15 iterations are sufficiently large to recover the

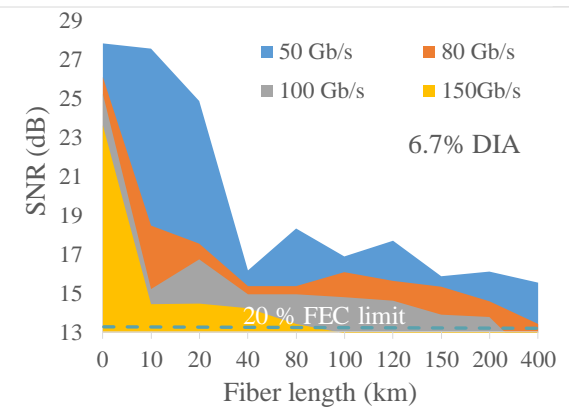

(a)

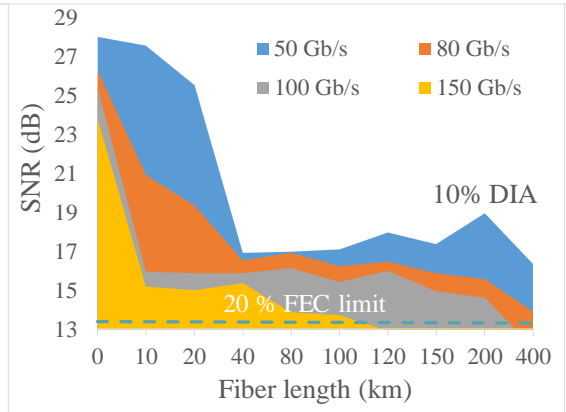

(b)

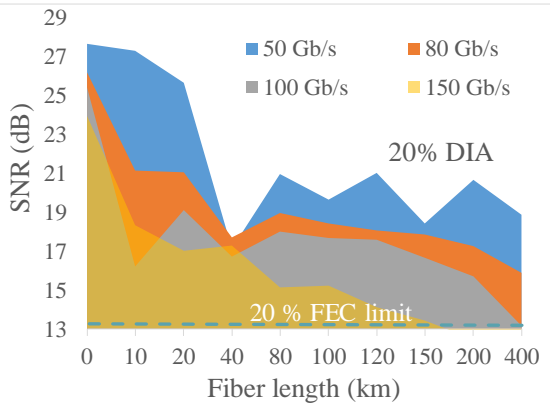

(c)

Fig. 6. The SNR of the recovered signal by DIA at different data rates and transmission distances. The training overheads are (a) $6.7 \%$, (b) $10 \%$, and (c) $20 \%$. 
(a)
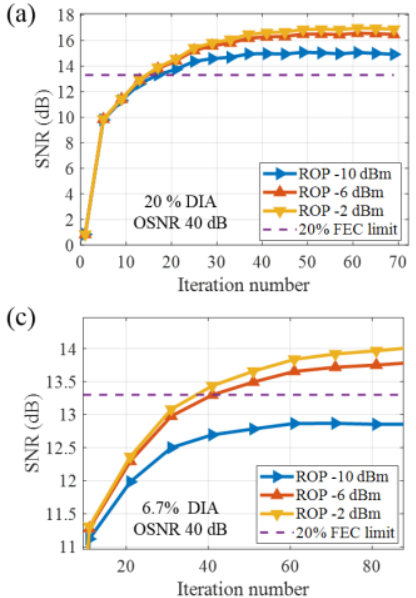

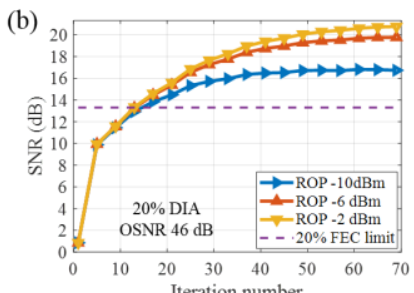

(d)

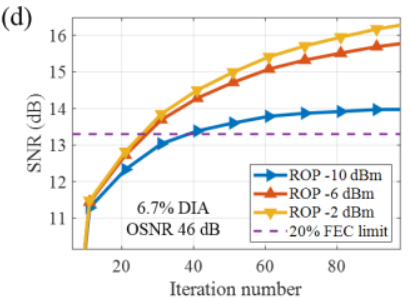

Fig. 7. The SNR of the recovered signal after different times of iteration. (a) 20\% overhead@40 dB OSNR; (b) 20\% overhead@46 dB OSNR; (c) 6.7\% overhead@40dB OSNR; (d)6.7\% overhead@46dB OSNR.

signal at the $20 \%$ FEC limit. The minimum required iterations are almost the same for different ROPs. However, for $6.7 \%$ DIA, some convergence speed differences appear for different ROPs and OSNRs. At the 20\% FEC limit, the minimum number of iterations is 40 for the considered cases. This demonstrates that our training strategy can accelerate the convergence speed of conventional IA and dramatically improves its retrieval ability. Moreover, all the curves monotonically increase with iteration number, this verifies the DIA algorithm stability.

\section{Parameter Optimization}

The reconstructed signal performance of the proposed DIA technique is closely related to the following parameters such as amplitude offset, iteration interval and pilot overhead. Here the amplitude offset is defined as the direct-current (DC) component of a PD-detected optical current. This parameter should be estimated before the iteration process starts. In this subsection, we show the impacts of the first two factors on the transmission performance of $100 \mathrm{~Gb} / \mathrm{s}$ PAM4 signals over a 50 $\mathrm{km}$ SSMF IM/DD transmission system at an OSNR of $43 \mathrm{~dB}$ (ROP of $0 \mathrm{dBm}$ ).

We first sketch the SER versus the offset of the estimated DC component $\eta$ in Fig. 8(a), where $\eta=\left(V_{\text {adopt }}-V_{\text {true }}\right) / V_{\text {true }} \times 100 \%$, $V_{\text {adopt }}$ is the estimation of the real amplitude of the DC component $V_{\text {true }}$. As shown in Fig. 8(a), a DIA with a positive DC offset suffers less performance degradations than a negative DC offset, this indicates that we can choose a relative larger DC offset when a slight performance degradation is allowed.

We then depict the DIA performance versus iteration interval illustrated in Fig. 2 in Fig. 8(b). It is shown that the SER decreases with decreasing iteration interval. To obtain lower SERs, a pilot insertion needs to be applied at each cycle of the iteration, where the iteration interval equals 1. Because Eq. (10) is simpler than Eq. (8), and the SER rises slowly as a function of iteration interval, as such the iteration interval can be increased appropriately to obtain an optimum trade-off between the SER and the complexity. The results shown here are

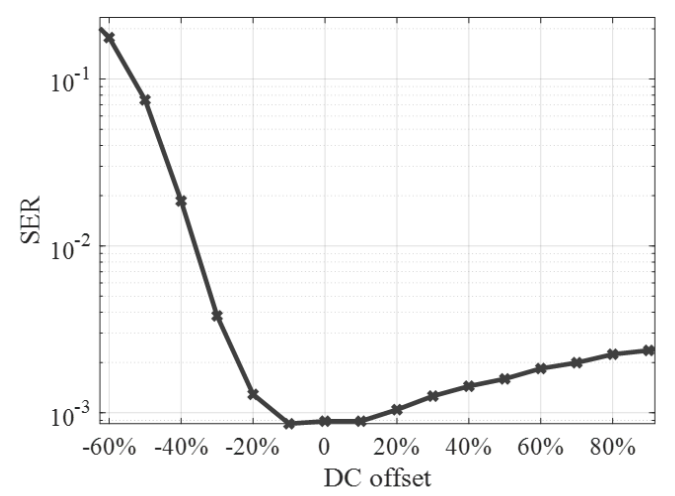

(a)

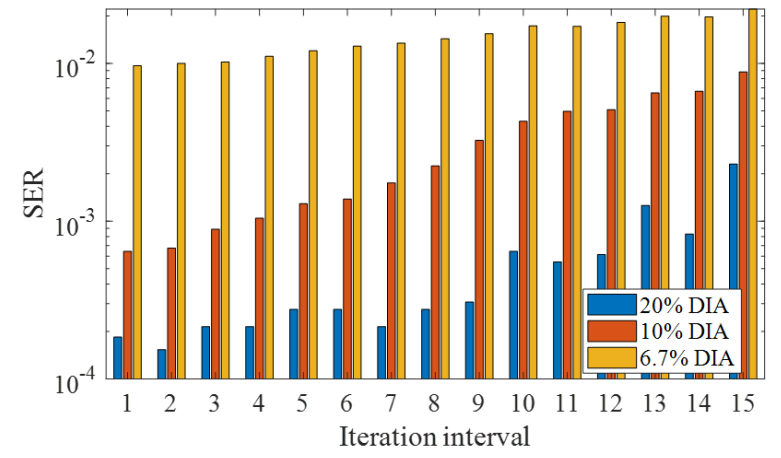

(b)

Fig. 8. The SER curve and bar graph versus (a) the proportion of the DC offset and (b) the iteration interval.

obtained for an iteration interval of 3. We also find in Fig. 8(b) that even the SER decreases when the overhead increases, we cannot, however, increase the overhead discretionarily. In the next section, we introduce a method to increase the number of pilot symbols but without increasing the overhead.

\section{DECISION-DIRECTED DIA FOR CONVERGENCE ACCELERATION}

In this section, we propose a modified DIA with higher performance named DD-DIA and verify its effectiveness. In this algorithm, we introduce a decision processes into DIA to generate more pilot symbols but without increasing the overhead for the first time. The algorithm is described below:

Phase 1, DIA stage: using $P \times$ iterations of DIA for pre-convergence to guarantee the effectiveness of Phase 2;

Phase 2, decision-directed (DD) stage: from the $(P+1)$-th iteration, a decision block is inserted after the match filter to decide the transmitted symbol $\mathrm{D}[x(n)]$. We use a random variable $\theta(n, k)$ which follows the Bernoulli distribution at the $n$-th symbol and the $k$-th iteration, as shown in Eq. (11).

$$
\operatorname{Pr}\{\theta=1\}=p, \operatorname{Pr}\{\theta=0\}=1-p, p \in[0,1],
$$

where "Pr" stands for probability. Then the symbols after hard decision is randomly selected to substitute the signal $x(n)$, as expressed in Eq. (12)

$$
\mathrm{G}^{\prime}\{x(n)\}=\theta(n, k) \mathrm{D}[x(n)]+[1-\theta(n, k)] x(n) .
$$

Afterwards, we use the pilot symbols $d_{\text {pilot }}(n)$ to replace the obtained signal $\mathrm{G}^{\prime}\{x(n)\}$ as described in Eqs. $(13,14)$ 
Totally 200 times iterations Overhead ratio of training symbol is $10 \%$

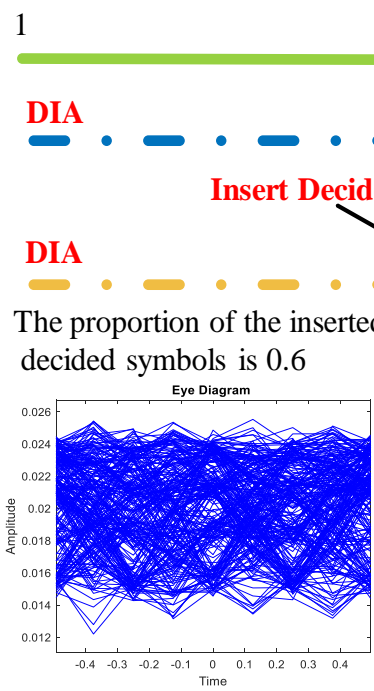

(b)
120

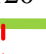

200

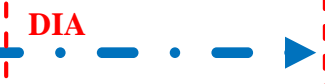

ided Data DD-DIA

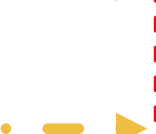

(a)

Iteration index

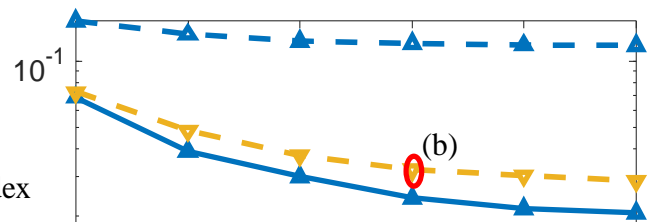

$\frac{\alpha}{5}$

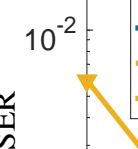

DD-DIA $100 \mathrm{~Gb} / \mathrm{s} 400 \mathrm{~km} 43 \mathrm{~dB}$ OSNR

- DIA $100 \mathrm{~Gb} / \mathrm{s} 400 \mathrm{~km} 43 \mathrm{~dB}$ OSNR

-DD-DIA $100 \mathrm{~Gb} / \mathrm{s} 100 \mathrm{~km} 40 \mathrm{~dB}$ OSNR

= DIA $100 \mathrm{~Gb} / \mathrm{s} 100 \mathrm{~km} 40 \mathrm{~dB}$ OSNR

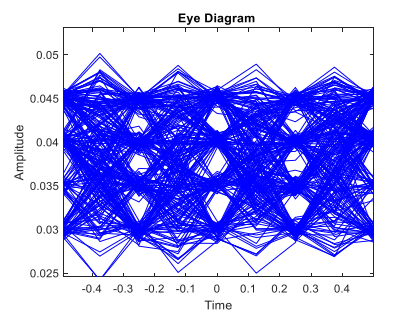

(c)

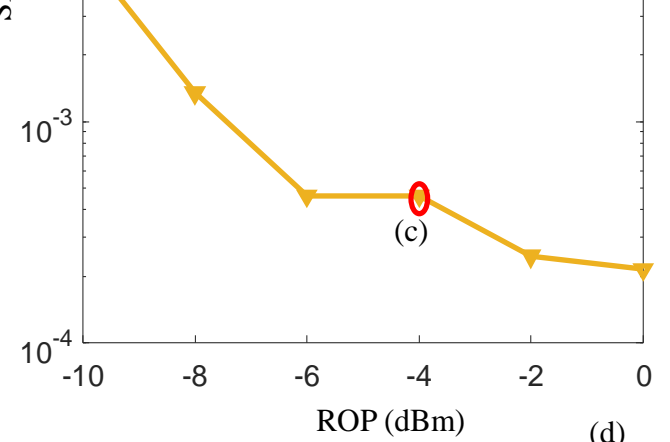

Fig. 9 Illustration of DD-DIA the signal reconstruction performance comparison between DIA and DD-DIA. The eye diagrams in subgraphs (b) and (c) correspond to the SER-ROP points circled in subgraph (d).

$$
\begin{aligned}
& \mathrm{G}\{x(n)\}=\mu_{M, L}(n, k) d_{\text {pilot }}(n)+\left[1-\mu_{M, L}(n, k)\right] \mathrm{G}^{\prime}\{x(n)\}, \\
& \mu_{M, L}(n, k)= \begin{cases}1, & \text { for } \bmod (n, M)=0 \& \bmod (k, L)=0 \\
0, & \text { otherwise }\end{cases}
\end{aligned}
$$

We name the decided symbols $\theta(n, k) \mathrm{D}[x(n)]$ as pseudo-pilot symbols because they are not the true pilot symbols but have a function similar to true pilot symbols when the decision is correct. The "random" selection of the pseudo-pilot symbol can further accelerate the convergence performance since different combinations of pseudo-pilot symbols in different iterations offer a variety of reference signals for DIA to ensure the stable convergence.

Figure 9 (a) illustrates the main difference between DIA and DD-DIA. The blue and yellow dash-dotted line represents the DIA mentioned in Section II and DD-DIA discussed in this section respectively. In this example, $P=120$ and $p=0.6$ for decided symbol random allocation. The iteration interval $L=3$ and the overhead is $10 \%$.

In Fig. 9(d), the numerically simulated results of the SERs versus ROP for DIA and DD-DIA are compared in $100 \mathrm{~Gb} / \mathrm{s}$ over $100 \mathrm{~km}$ and $400 \mathrm{~km}$ DSB IM/DD PAM4 transmission systems. The total number of iterations of DIA and DD-DIA are 200. In comparison with DIA, for $100 \mathrm{~Gb} / \mathrm{s}$ over $100 \mathrm{~km}$ optical transmissions, the SER of DD-DIA has 2 orders of magnitude reduction. For a $400 \mathrm{~km}$ transmission distance, the SER drops below the $20 \%$ FEC limit at $-8 \mathrm{dBm}$ ROPs, where the SER is dropped from 0.15 to $4 \times 10^{-2}$ (for Gray coding, the corresponding BER of $2 \times 10^{-2}$ is the $20 \%$ FEC limit). This signal reconstruction approach outperforms Volterra equalization and conventional IA and DIA, because: (1) we are fully utilizing the real character of the modulation type and the prior knowledge of the transmission system; (2) the convergence stability, global optimum feature and convergence speed are improved step by step with the aid of pilot symbols and pseudo-pilot symbols. For $100 \mathrm{~Gb} / \mathrm{s}$ PAM4 over 100-km SSMF transmission at the ROP of $-4 \mathrm{dBm}$, comparing between Fig. 9(b) and (c), it is found that the DD-DIA's eye diagram is almost fully recovered, this benefits from the decision-introduced convergence acceleration.

To compare the convergence performance between DIA and DD-DIA, we sketch their SER curves versus iteration index for different transmission distances in Fig. 10. For DD-DIA, the decision process is enabled after the 120-th iteration, this results in the disappearance of the error floor that occurs in DIA. The DD-DIA algorithm gives a rapid SER reduction especially when the SER of DIA is not sufficiently high. When the SER of DIA is up to more than $1 \times 10^{-1}$, we still can observe the SER reduction from DD-DIA, as shown in the blue curves of the $400-\mathrm{km}$ case.

Since an optimum selection of the starting iteration of the decision process in Phase 2 can obtain a better performance with a lower total iteration number, we depict SER curves versus iteration index in Fig. 11, where Phase 2 begins at different iterations for $100 \mathrm{~Gb} / \mathrm{s}$ PAM4 signals over $200 \mathrm{~km}$ SSMF transmissions. The OSNR and ROP are kept at $40 \mathrm{~dB}$ and $-4 \mathrm{dBm}$, respectively. From Fig. 11, we can find that the decision process can reduce the SER when the SERs of DIA are lower than 0.1. To achieve an optimum SER performance, the activation time of the DD block is suggested to set when the SER is lower than $6 \times 10^{-2}$, as shown in the blue curve in Fig. 11 . The DD-DIA is capable of reducing the SER below the $20 \%$ and $7 \%$ FEC limit within only 25 and 60 iterations, which saves more time and reduces the computational complexities compared to the "error escape" method in [13]. 


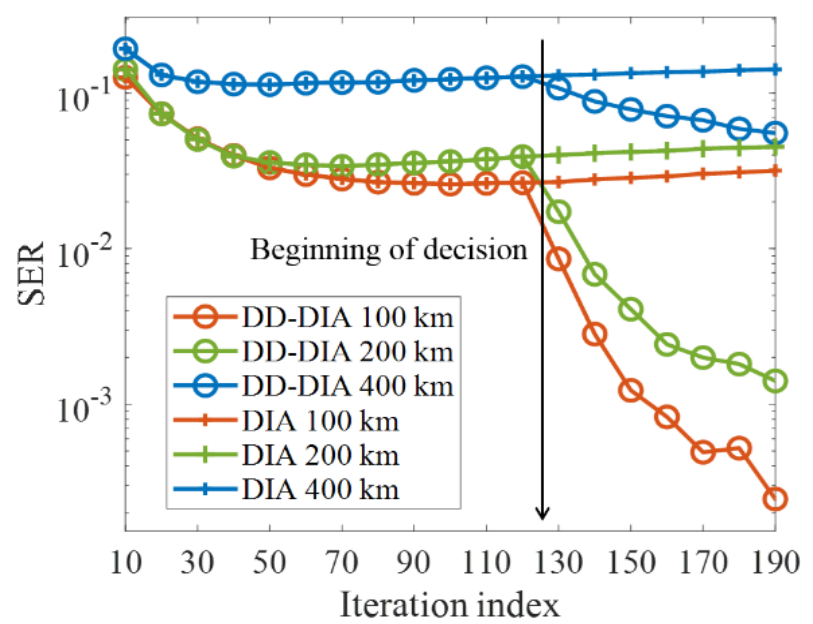

Fig. 10. SER versus number of iteration for DIA and DD-DIA calculated at 40 $\mathrm{dB}$ OSNR and $-4 \mathrm{~dB}$ ROP. Decision is enabled from the 121th iteration.

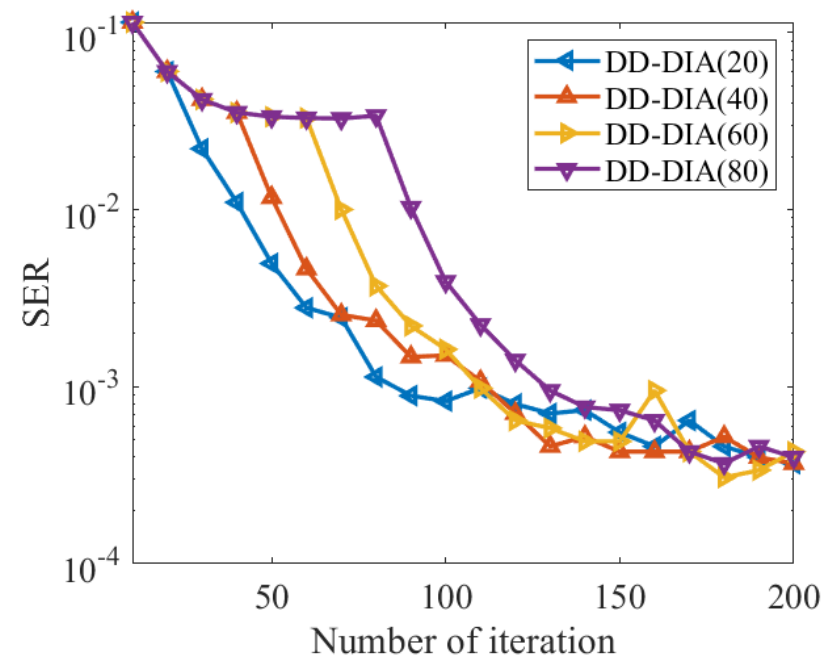

Fig. 11. SER versus the number of iteration cycles of DD-DIA. The legend "DD-DIA (20/40/60/80)" represents the starting point of decision is activated from the $20^{\text {th }} / 40^{\text {th }} / 60^{\text {th }} / 80^{\text {th }}$ cycle of iteration. The $100 \mathrm{~Gb} / \mathrm{s}$ PAM 4 signal is transmitted over $200 \mathrm{~km} \mathrm{SSMF}$ at the OSNR and ROP of $40 \mathrm{~dB}$ and $-4 \mathrm{dBm}$.

To explore the tolerance of DIA and DD-DIA to dispersion parameter mismatch between the real value and the estimated value, numerical simulations are conducted where the dispersion mismatch is represented by a fiber length mismatch in kilometer. For both DIA and DD-DIA, the results are plotted in Fig. 12 as a function of fiber length mismatch. Note that ideal optical amplifiers are adopted in the simulations. With the help of the decision process, we find that no performance degradations occur when the fiber length increases from $40 \mathrm{~km}$ to $100 \mathrm{~km}$ at an OSNR of $40 \mathrm{~dB}$. At a SER of $1 \times 10^{-2}$ for the 40 $\mathrm{km}$ SSMF transmission, DIA has $1 \mathrm{~km}$ fiber length estimation error tolerance at an OSNR of $46 \mathrm{~dB}$, while DD-DIA doubles the tolerance of DIA (dynamic range is 5\%). For the $100 \mathrm{~km}$ fiber transmission, the tolerance is similar to that corresponding to $40 \mathrm{~km} \mathrm{SSMF}$, this reveals that a higher estimation accuracy is required (2\%) for a longer fiber distance. When there is a dispersion deviation, it is equivalent to apply a perturbation to the propagation equation. The DIA and DD-DIA have a finite error correction capability, so a moderate perturbation results in their performance degradation while a large perturbation makes

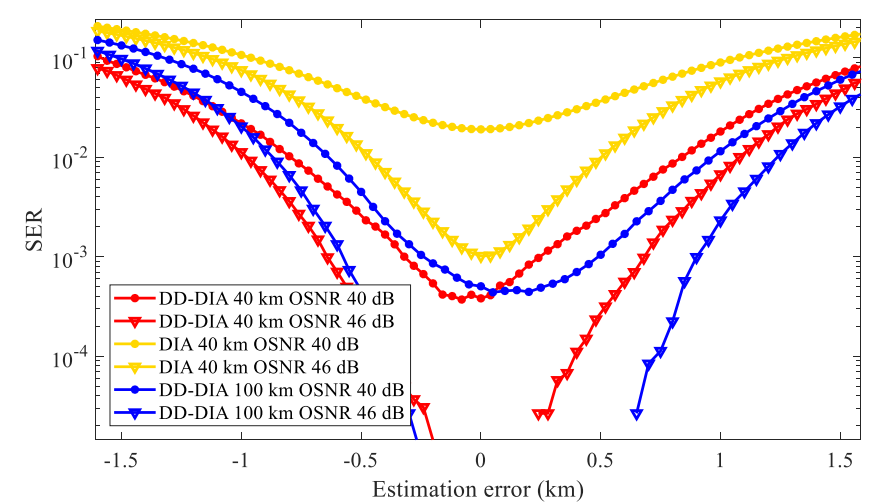

Fig. 12. SER versus the estimation error of fiber length for $100 \mathrm{~Gb} / \mathrm{s}$ PAM4 DSB IM/DD system. The ROP is $-4 \mathrm{dBm}$. The starting beginning cycle index of iteration of DD-DIA is 80 . The total iteration number is 200 .

them insufficient to align the signal values at each iterations, and consequently, this perturbation accumulates, step by step, calculation errors step over step-into the signal to be retrieved, which finally causes the performance divergence.

To accurately estimate the dispersion parameter and other parameters mentioned above, we can use two methods in practice: the first one is a recursive method that is based on the fast modelling technique of machine learning, typically for transfer learning with deep neural network. The other one is to use an optimization method by parameter sweeping or searching. Based on the knowledge of channel characteristics, DIA and DD-DIA can support the optical field reconstruction in IM/DD system with a higher transmission capacity.

To accurately estimate the dispersion parameter and other parameters mentioned above, we can use two methods in practice: the first one is a recursive method that is based on the fast modelling technique of machine learning, typically for transfer learning with deep neural network. The other one is to use an optimization method by parameter sweeping or searching. Based on the knowledge of channel characteristics, DIA and DD-DIA can support the optical field reconstruction in IM/DD system with a higher transmission capacity.

\section{CONCLUSION}

We have proposed and investigated two novel data-aided iterative algorithms termed DIA and DD-DIA for linearizing IM/DD optical transmission systems. DD-DIA is a promising algorithm for reconstructing optical fields of intensity modulated and directly detected signals with the following advantages, (1) No modifications to hardware structures; (2) high transmission capacity; (3) fast convergence speeds (within 50 cycles of iterations) without "escape" re-initialization; (4) large tolerance to errors in channel parameter estimation, and (5) significant OSNR/ROP gains. This refreshes our knowledge of the transmission extreme of IMDD transmission systems, our results indicate that the proposed technique has great potential for practical implementation. 


\section{REFERENCES}

[1] K. Kikuchi, "Fundamentals of Coherent Optical Fiber Communications," J. Lightwave Technol., vol. 34, no. 1, pp. 157-179, 2016.

[2] X. Ruan, L. Zhang, F. Yang, Y. Zhu, Y. Li, and F. Zhang, "Beyond 100G Single Sideband PAM-4 Transmission With Silicon Dual-Drive MZM," IEEE Photon. Technol. Lett., vol. 31, no. 7, pp. 509-512, 2019.

[3] A. Mecozzi, C. Antonelli, and M. Shtaif, "Kramers-Kronig coherent receiver," Optica, vol. 3, no. 11, pp. 1220-1227, 2016.

[4] X. Li et al., "Transmission of $4 \times 28-\mathrm{Gb} / \mathrm{s}$ PAM-4 over $160-\mathrm{km}$ single mode fiber using 10G-class DML and photodiode," in 2016 Optical Fiber Communications Conference and Exhibition (OFC), 2016, pp. 1-3.

[5] D. Che, C. Sun, and W. Shieh, "Maximizing the spectral efficiency of Stokes vector receiver with optical field recovery," Opt. Express, vol. 26, no. 22, pp. 28976-28981, 2018.

[6] S. An, Q. Zhu, J. Li, and Y. Su, "Accurate Field Reconstruction at Low CSPR Condition Based on a Modified KK Receiver With Direct Detection," J. Lightwave Technol., vol. 38, no. 2, pp. 485-491, 2020.

[7] N. Stojanovic, F. Karinou, Z. Qiang, and C. Prodaniuc, "Volterra and Wiener Equalizers for Short-Reach 100G PAM-4 Applications," $J$. Lightwave Technol., vol. 35, no. 21, pp. 4583-4594, 2017.

[8] H. Xin et al., "Nonlinear Tomlinson-Harashima precoding for direct-detected double sideband PAM-4 transmission without dispersion compensation," Opt. Express, vol. 27, no. 14, pp. 19156-19167, 2019.

[9] J. Zhou et al., "Joint FDE and MLSD Algorithm for 56-Gbit/s Optical FTN-PAM4 System Using 10G-Class Optics," J. Lightwave Technol., vol. 37, no. 13, pp. 3343-3350, 2019.

[10] G. Goeger, C. Prodaniuc, Y. Ye, and Q. Zhang, "Transmission of intensity modulation-direct detection signals far beyond the dispersion limit enabled by phase-retrieval," in 2015 European Conference on Optical Communication (ECOC), 2015, pp. 1-3.
[11] A. S. Karar, "Iterative Algorithm for Electronic Dispersion Compensation in IM/DD Systems," J. Lightwave Technol., vol. 38, no. 4, pp. 698-704, 2020.

[12] G. Goeger, "Applications of Phase Retrieval in High Bit-Rate Direct-Detection Systems," in Optical Fiber Communication Conference, Anaheim, California, pp. Th2A.40, 2016.

[13] H. Chen, N. K. Fontaine, J. M. Gene, R. Ryf, D. T. Neilson, and G. Raybon, "Dual Polarization Full-Field Signal Waveform Reconstruction Using Intensity Only Measurements for Coherent Communications," $J$. Lightwave Technol., vol. 38, no. 9, pp. 2587-2597, 2020.

[14] T. L. Koch and R. A. Linke, "Effect of nonlinear gain reduction on semiconductor laser wavelength chirping," Appl. Phys. Lett., vol. 48, no. 10, pp. 613-615, 1986.

[15] C.-C. Wei, "Analysis and iterative equalization of transient and adiabatic chirp effects in DML-based OFDM transmission systems," Opt. Express, vol. 20, no. 23, pp. 25774-25789, 2012.

[16] Y. Gao et al., "40 Gb/s CAP32 short reach transmission over $80 \mathrm{~km}$ single mode fiber," Opt. Express, vol. 23, no. 9, pp. 11412-11423, 2015.

[17] X. Gong, L. Guo, Y. Dong, M. Deng, R. P. Giddings, and J. M. Tang, "SPM-Improved Transmission Performance of Software-Reconfigurable IMDD PONs Based on Digital Orthogonal Filtering," J. Lightwave Technol., vol. 35, no. 20, pp. 4488-4496, 2017.

[18] S. Hu, X. Yi, J. Zhang, Y. Song, M. Zhu, and K. Qiu, "Volterra equalization of complex modulation utilizing frequency chirp in directly modulated lasers," Opt. Commun., vol. 409, pp. 99-104, 2018.

[19] H. Wang et al., "Adaptive Channel-Matched Detection for C-Band \begin{tabular}{llllll}
$64-G b i t / s$ & Optical & OOK & System & Over & $100-\mathrm{km}$ \\
\hline
\end{tabular} Dispersion-Uncompensated Link," J. Lightwave Technol., vol. 38, no. 18 , pp. 5048-5055, 2020. 